Research Article

\title{
Prematch Emotions and Coping Styles of Martial Arts Athletes Based on Artificial Intelligence
}

\author{
Anping Li, ${ }^{1}$ Hongfei Wu ${ }^{D},{ }^{2}$ and Yuan Liu ${ }^{3}$ \\ ${ }^{1}$ Department of Physical Education and Research, Yang-En University, Quanzhou 362014, Fujian, China \\ ${ }^{2}$ The School of Physical Education, Henan Polytechnic University, Jiaozuo 454000, China \\ ${ }^{3}$ Public Sports Teaching and Research Department, Luoyang Normal University, Luoyang 471000, Henan, China \\ Correspondence should be addressed to Hongfei Wu; wuhongfei@hpu.edu.cn
}

Received 26 May 2021; Revised 22 June 2021; Accepted 7 July 2021; Published 20 July 2021

Academic Editor: Sang-Bing Tsai

Copyright (C) 2021 Anping Li et al. This is an open access article distributed under the Creative Commons Attribution License, which permits unrestricted use, distribution, and reproduction in any medium, provided the original work is properly cited.

\begin{abstract}
The fluctuation of martial arts athletes before competition is easy to affect martial arts competition. Emotional stimulation helps martial arts athletes to play in the competition field and improve their on-the-sport coping skills. In this paper, we use artificial intelligence technology to analyze the precompetition emotions of martial arts athletes, make guidance for the precompetition situation of martial arts athletes, and give to-the-spot response guidance and suggestions. In this paper, the artificial intelligence MLR (multiple logistic regression) combined with the unsupervised LOF (local outlier factor) algorithm is used to realize the precompetition emotion analysis and on-the-spot response guidance for martial arts athletes. In addition, this paper takes a community martial arts team as the test object and verifies the method through practice feedback. Studies have shown that this method of analysis and guidance can effectively assess the emotions of martial arts athletes and take intervention, compared to the absence of sentiment analysis and on-the-sport guidance for martial arts athletes. Compared to the coach's intuitive guidance, the interventions in this treatise speed up 65\%. From the experimental subjects in this paper, athletes of the same level provide emotional guidance and suggestions for responding to emotional fluctuations. This can psychologically improve the efficiency of athletes by more than $35 \%$. Therefore, the use of artificial intelligence analysis methods can effectively improve the emotional stability of martial arts athletes before the game, so that they can deal with all kinds of emergencies on the spot.
\end{abstract}

\section{Introduction}

Based on emotional event theory, decision-making participation is a typical internal learning behavior in an organization. In the process of interpersonal interaction, members of an organization will form a collective emotional response and individual work efficacy through emotional rendering, sharing, and aggregation mechanisms. The external performance of this emotional spiral mechanism needs the help of a specific decision-making participation process among members and then through the ability of resource integration to obtain a competitive advantage.

Some studies have extensively discussed the relationship between AI sentiment analysis and organizational innovation. For example, Zheng et al. found that AI sentiment analysis can promote non-family business innovation but can not significantly promote family business innovation [1].
Harreiter and Roden further pointed out that martial arts athletes' sense of self-efficacy and decision-making participation interact with performance; that is, when martial arts athletes have a sense of high efficiency, their decisionmaking participation can improve work performance [2]. Hackett and Steptoe further emphasize that the fault-tolerant dynamics of the organization encourage members to actively express their emotions and freely express taboo views. It is more necessary to realize the system implementation and resource collection and promote the performance improvement through the dialogue and brainstorming knowledge integration ability in decisionmaking [3]. Abrahamian et al. found that AI sentiment analysis is beneficial to product innovation of science and technology enterprises, thus improving performance [4]. On this basis, Sartorius puts more emphasis on the importance of AI sentiment analysis and organizational innovation 
environment and believes that only appropriate supportive environments can promote organizations to search for knowledge, grab resources from the outside, and learn from the success or failure of risks, to obtain business innovation opportunities [5].

On the spot of martial arts athletes, Petersmann et al. proposed fault tolerance and experimental environment modeling of fault tolerance dynamics, which provides a supportive environment for AI emotional analysis and organizational innovation [6]. Arneth et al. believe that fault tolerance dynamics itself contains the ability of the organization to stimulate martial arts athletes' interests, creative ideas, and innovative consciousness by creating a fault tolerance environment, and the theory of dynamic ability regards such ability as the key to achieving the balance of organizational performance [7]. Bryan proposed that organizational fault tolerance legitimizes random, uncensored experiments and errors. When free experiments become feasible, creativity emerges from AI emotion analysis [8]. Hägerhäll et al. proposed that the test environment in fault tolerance dynamics can promote the members of the organization to take risks and try first and realize the scheme customization by trying new ideas and improving new processes [9]. Hand and Brown pointed out that when the new idea, experiment, or beneficial attempt of the organization members gets the positive response from the organization, it is easier for them to invest resources in product development and process improvement, to achieve high performance [10]. The above research is based on the path of convergence. During the decision-making process, members of the organization actively express their opinions, influence their decisions, and recognize the importance of their opinions. This will undoubtedly increase participation in innovation activities and force members to rebuild and improve their knowledge systems. It strengthens the balance between their ability to innovate and performance through the convergence of values. However, there is a lack of analysis of on-the-spot coping style and prematch emotions.

In this paper, we use artificial intelligence technology to analyze the precompetition emotions of martial arts athletes, make guidance for the precompetition situation of martial arts athletes, and give to-the-spot response guidance and suggestions. In this paper, the artificial intelligence MLR (multiple logistic regression) combined with the unsupervised LOF (local outlier factor) algorithm is used to realize the precompetition emotion analysis and on-the-spot response guidance for martial arts athletes. In addition, this paper takes a community martial arts team as the test object and verifies the method through practice feedback.

\section{Martial Arts Skills Promotion and AI Logistic Regression Emotion Classification Algorithm}

2.1. AI Martial Arts Teaching Methods. The application of artificial intelligence education has brought about the epistemological transformation of the teaching knowledge of martial arts coaches. At present, the application of artificial intelligence education has challenged the development of the teaching knowledge of traditional martial arts coaches and contributed to the epistemological transformation of the teaching knowledge of martial arts coaches; it is mainly reflected in the main components of martial arts coaches' subject teaching knowledge, which should be expanded from martial arts coaches' teaching and students' learning of artificial intelligence teaching and learning [11]. However, under the background of the current application of artificial intelligence education, due to the extensive and deep integration of artificial intelligence teaching and aid systems, and the substitution of artificial intelligence for part of the knowledge teaching work of martial arts coaches, martial arts coaches not only need to master the algorithm logic and data logic behind artificial intelligence, but also need to master the teaching design, implementation, and implementation based on artificial intelligence to manage the construction and evaluation of the subject teaching knowledge (AI emotion analysis) integrating AI with martial arts coaches; it is necessary to master the relevant knowledge of effective teaching with the help of AI [12]. At the same time, based on the rapid development of artificial neural networks and deep learning, artificial intelligence itself "can find the complex structure in high-dimensional data and achieve better results than traditional machine learning methods." Therefore, martial arts coaches should also master the learning process of artificial intelligence and make the learning evolution trajectory of artificial intelligence clear [13]. Therefore, under the background of the current application of artificial intelligence education, the composition of teaching knowledge of martial arts coaches has changed. Martial arts coaches not only need to master the teaching representation and strategy knowledge based on students' understanding, but also need to master the corresponding knowledge of artificial intelligence teaching and learning and make the knowledge logic behind artificial intelligence teaching and learning clear [14]. The dimension of teaching knowledge of martial arts coaches should be from a single cognitive dimension to the multidimensional attention of cognition, emotion, and morality [15]. Background of the current application of artificial intelligence in education is based on the current development stage of artificial intelligence technology and human centricity, the principles of artificial intelligence development, the regulation of the algorithm model behind artificial intelligence, and the promotion of educational big data. In place of the martial arts coach, it performs educational interactions with students but can only perform corresponding cognitive interactions with students, and the cognitive interactions between AI and students are predominant. It is a low-level interaction that focuses solely on cognitive outcomes [16]. People can only be educated through people who have received the same education. Due to the current development stage of weak artificial intelligence, although artificial intelligence has certain emotion recognition ability, it is difficult to reach emotional resonance with students [17]. Therefore, in the current development process of martial arts coaches' subject teaching knowledge, martial arts coaches should not only pay attention to the specific teaching representation and strategies of subject content knowledge based on students' understanding, but also pay attention to 
the emotional state and moral development of students' learning; learning based on students' subject knowledge promotes students' moral development [18]. Therefore, under the background of the current application of artificial intelligence education, the dimension of teaching knowledge of martial arts coaches has changed from the traditional single cognitive dimension to the multidimensional attention of cognition, emotion, and morality. The teaching knowledge level of martial arts coaches should move from the development of students' low-level cognition to the integrated development of high-level teaching and education [19]. Therefore, the high-level subject knowledge teaching of martial arts coaches mainly points to the complex and open teaching, which is student-centered and promotes the development of students' critical thinking. It is no longer limited to martial arts coaches imparting rote knowledge to students (which has been replaced by artificial intelligence) but requires martial arts coaches to use artificial intelligence to teach and learn on the basis of students' learning and development psychology, subject knowledge development logic, and corresponding social development requirements; in the specific education and teaching situation, the knowledge can be understood by students. The way of thinking is presented to students, and students are organized to carry out corresponding reflections and discussions, to promote students' understanding, criticism, and application of knowledge [20]. At the same time, due to the nature of the development of educational teaching and the fact that artificial intelligence is hard to replace the work of martial arts coaches' subject education, the high-level subject knowledge teaching of martial arts coaches should be the unity of "teaching and educating people." Martial arts coaches should not only pay attention to the high-level learning of students' subject knowledge, but also pay attention to the infiltration and teaching of moral education knowledge, socialist core values, and excellent traditional culture. They should pay more attention to the ethics of their own teaching process, "from good to good," and combine teaching with educating people, to move towards the integrated development of high-level teaching and educating people [21]. Therefore, under the background of the current application of artificial intelligence education, the teaching knowledge level of martial arts coaches should change from the development of students' low-level cognition to the integration of high-level teaching and education. In the preparation stage before the competition, it can replace the martial arts coach to help students prepare and lead students to master the basic elementary knowledge [22]. In the homework stage after class, it can replace the martial arts coach to correct students' homework and guide students to review the corresponding knowledge. Due to the large knowledge engineering support behind artificial intelligence and the promotion of its own deep learning, the knowledge learning ability and general knowledge teaching ability of artificial intelligence are far better than human beings [23]. As a result, artificial intelligence can replace part of the traditional knowledge teaching work of martial arts coaches, which also promotes the development of higher-level teaching knowledge of martial arts coaches. Therefore, to deal with the survival crisis and professional development dilemma brought by artificial intelligence to martial arts coaches and to establish the new characteristics of martial arts coaches' professional development under the background of artificial intelligence education application, the level of martial arts coaches' subject teaching knowledge needs to be upgraded [24]. Artificial intelligence for martial arts coaches to save the necessary labor time will correspondingly broaden the time and space of professional development of martial arts coaches, to promote the transformative development of martial arts coaches' subject teaching knowledge. With the partial replacement and liberation of AI for martial arts coaches' knowledge teaching, martial arts coaches are forced to incorporate AI into their own subject knowledge teaching process to ensure the integration and guidance of martial arts coaches for AI teaching, but martial arts coaches also get the corresponding time and energy, can pay more attention to the emotional interaction between themselves and students, and can carry out the integrated teaching of high-level subject knowledge based on the low-level subject knowledge teaching of artificial intelligence. Artificial intelligence has its advantages and disadvantages in the field of education. Because of the particularity of artificial intelligence, martial arts coaches integrate the subject teaching knowledge of artificial intelligence (artificial intelligence, emotion analysis) with its unique connotation characteristics and structural model.

\subsection{Multiclass Logistic Regression Emotion Classification} Algorithm. Precompetition trauma can significantly increase the possibility of individuals entering high aggressive and high negative emotions (martial arts tactics I) and low aggressive and high negative emotions (martial arts tactics III):

$$
\begin{aligned}
P^{(k)} & =\partial_{1} l_{\mathrm{bce}}^{(k)}+\partial_{2} l_{\mathrm{iou}}^{(k)}+\partial_{3} l_{\mathrm{ssim}}^{(k)}, \\
I & =\frac{n \sum_{i=1}^{n} \sum_{j=1}^{n} w_{i j}\left(x_{i}-\bar{x}\right)(x j-\bar{x})}{\sum_{i=1}^{n} \sum_{j=1}^{n} w_{i j}(x i-\bar{x})^{2}}=\frac{n \sum_{i=1}^{n} \sum_{i \neq j}^{n} w_{i j}\left(x_{i}-\bar{x}\right)(x j-\bar{x})}{S^{2} \sum_{i=1}^{n} \sum_{j=1}^{n} w_{i j}}, \\
w & =1-\frac{\sum_{a=1}^{H} \sum_{b=1}^{W} \operatorname{SEG}(a, b) \mathrm{GT}(x i-\bar{x})}{\sum_{a=1}^{H} \sum_{b=1}^{W}[\operatorname{SEG}(x i-\bar{x})+G T(x i-\bar{x})-\operatorname{SEG}(x i-\bar{x}) \mathrm{GT}(x i-\bar{x})]}
\end{aligned}
$$


Emotional neglect and physical neglect are independent risk factors of high aggressive and negative emotions (martial arts tactics I); emotional neglect is an independent risk factor that significantly increases the individual's entry into low aggression and high negative emotion (martial arts tactics III):

$$
M_{1}=\left\{\begin{array}{l}
s-p_{1}-x_{1}, \\
x-p_{2}-\left(1-x_{1}\right) .
\end{array}\right.
$$

According to the number of different types of precompetition trauma, the cumulative effects between different types of precompetition trauma and emotional behavior subtypes were further explored:

$$
\begin{aligned}
P * & =\beta *\left(\frac{L}{(A)+(\operatorname{In}-\alpha W)^{-1} \varepsilon}\right), \\
\mathrm{LCA}_{\kappa} & =\frac{2 k}{k+1}+\left[\frac{1}{2}+\frac{1}{2 k}\right]\left[\frac{c_{2}-c_{1}}{3}\right]^{2}+\frac{2\left(c_{2}-c_{1}\right)}{3} .
\end{aligned}
$$

The more trauma types and individual experiences before competition, the more likely he is to enter into high aggressive and high negative emotions (martial arts tactics). Then four different subtypes of emotional behavior were identified by LCA:

$$
\begin{aligned}
\frac{(\operatorname{In}-\alpha W)}{\mathrm{LCA}} y & =(\operatorname{In}-\alpha W) X \beta+\varepsilon, \\
P\left(d_{i}, w_{j}\right) & =P\left(d_{i}\right) P\left(w_{j} \mid d_{i}\right), \\
P\left(w_{j} \mid d_{i}\right) & =\sum_{k=1}^{K} P\left(w_{j} \mid z_{k}\right) P\left(z_{k} \mid d_{i}\right), \\
l_{\text {ssim }} & =1-\frac{\left(2 \mu_{x} \mu_{y}+C_{1}\right)\left(2 \sigma_{x y}+C_{2}\right)}{\left(\mu_{x}^{2}+\mu_{y}^{2}+C_{1}\right)\left(\sigma_{x}^{2}+\sigma_{y}^{2}+C_{2}\right)} .
\end{aligned}
$$

In particular, we need to pay attention to individuals with high aggressive and negative emotions. In addition, this study pointed out that the identification of precompetition trauma is an important measure to prevent emotional behavior problems of higher vocational nursing students, especially to pay more attention to individuals with emotional neglect experience. With the subdivision of labor between artificial intelligence and humans and the surge in the landing of artificial intelligence in various industries in the field of education, artificial intelligence is used to promote the development of modernization of education and promoting educational reform and academia consensus

$$
\begin{aligned}
\ln \left(\mu_{x} \mu_{y}+C\right)_{i t} & =a_{0}+a_{1} \mathrm{~d} u * \mathrm{~d} t+\sum_{i=1}^{N} b_{j} X u+\varepsilon_{u}, \\
\mathrm{SEN} & =\frac{|\mathrm{SEG} \cap \mathrm{GT}|}{\mathrm{GT}}, \\
\theta(p, q) & =\arctan \left(\frac{L(p, q+1)-L(p, q-1)}{L(p+1, q)-L(p-1, q)}\right) .
\end{aligned}
$$

Due to the partial substitution of artificial intelligence for the role of traditional martial arts coaches, the basic subject teaching knowledge of traditional martial arts coaches' professional development is also facing new challenges, mainly reflected in the extensive and in-depth integration of artificial intelligence in the teaching field, and the technical components of traditional martial arts coaches' subject teaching knowledge are facing renewal:

$$
\begin{aligned}
S_{H} & =\frac{p_{2}-p_{1}+1}{2}, \\
P & =100 \% \times\left(1-\frac{|\mathrm{SEG}|-|\mathrm{GT}|}{|\mathrm{GT}|}\right) .
\end{aligned}
$$

Among them, artificial intelligence teaching system seg, tutor system GT, and educational robot are replacing part of the traditional knowledge teaching work of martial arts coaches. Relevant research points out that "on the whole, educational robots help to improve students' learning effects." Intelligent tutor system can significantly improve the students' athletes' emotion. The widespread application of information technology in education has transformed the subject education knowledge of martial arts coaches into subject education knowledge that integrates technology and subject education knowledge that makes good use of information and communication technology. However, "artificial intelligence as the commanding point of information technology," because of its deep learning, knowledge mapping technology, and the support of knowledge engineering, can gather and surpass the teaching advantages of traditional general information technology, so it is different from the traditional general technology. The technical components of traditional martial arts teaching knowledge are facing the renewal:

$$
\begin{aligned}
\text { Trend } & =\frac{2|I C T \cap L|}{|I C T|+|L|}, \\
y & =\alpha W y+\beta_{1} X-W \beta_{2} X+\varepsilon .
\end{aligned}
$$

Due to the cognitive interaction bias between teachers and students caused by artificial intelligence, the single cognitive dimension of traditional martial arts coaches' subject teaching knowledge needs to be expanded. Based on the education big data behind it, artificial intelligence can collect and analyze students' learning behavior data in the whole process and make visual digital portraits of students, to provide teaching decision-making suggestions for martial arts coaches. Thus, from the perspective of martial arts coaches, students are materialized into a group of data. Martial arts coaches mainly use artificial intelligence to carry out cognitive interaction with students from data to information to knowledge. Based on the support of knowledge mapping technology and knowledge engineering, artificial intelligence can correct students' homework anytime and anywhere and solve students' knowledge dilemmas in the learning process, to replace martial arts coaches to solve students' knowledge confusion. Therefore, from the student's point of view, the martial arts coach becomes an 
important component of the machine, and students can only perform cognitive interactions from data to information and knowledge with the martial arts coach through artificial intelligence. However, the traditional research on the subject teaching knowledge of martial arts coaches "pays too much attention to the cognitive dimension and ignores the emotional dimension and moral dimension." It mainly focuses on how martial arts coaches organize and represent the subject content knowledge as a form acceptable to students according to their interests and abilities:

$$
\begin{aligned}
\psi & =\sum_{x=1}^{\theta} V x=\sum_{x=1}^{9}\left(\frac{W x}{\sum_{1}^{n} W_{\mathfrak{J}}} S x\right), \\
U_{2} & =\left\{\begin{array}{l}
s-p_{1}-k x_{2}, \\
x-p_{2}-k\left(1-x_{2}\right),
\end{array}\right.
\end{aligned}
$$

where $x$ is the fluctuation difference index and $K$ is the number of iterations. Under the background of the current application of artificial intelligence education, to promote the multidimensional deep interaction between martial arts coaches and students from cognition to emotion to morality, the dimension of martial arts coaches' subject teaching knowledge needs to be expanded. Due to the substitution of artificial intelligence for the teaching of low-level subject knowledge of martial arts coaches, the level of subject teaching knowledge of traditional martial arts coaches needs to be upgraded. Shulman's original subject teaching knowledge pays more attention to how martial arts coaches teach knowledge to students in a way that students can understand. It pays attention to the difference between martial arts coach knowledge and subject expert knowledge but does not pay attention to the level of martial arts coach's subject teaching knowledge. Under the background of the current application of artificial intelligence education, in the design and development of the current artificial intelligence education and teaching system, martial arts coaches' teaching knowledge of low-level disciplines has been transferred to the artificial intelligence system [25].

\section{Experimental Design}

3.1. Research Methods. In this paper, we use artificial intelligence technology to analyze the precompetition emotions of martial arts athletes, make guidance for the precompetition situation of martial arts athletes, and give tothe-spot response guidance and suggestions. In this paper, the artificial intelligence MLR (multiple logistic regression) combined with the unsupervised lof (local outlier factor) algorithm is used to realize the precompetition emotion analysis and on-the-spot response guidance for martial arts athletes. In addition, this paper takes a community martial arts team as the test object and verifies the method through practice feedback.

3.2. Experimental Design. In this paper, in the generation of subject teaching knowledge of martial arts coaches integrating artificial intelligence, due to the different personality characteristics and teaching experience of different martial arts coaches, the structure of subject teaching knowledge generated by different martial arts coaches interacting with different teaching situations is different. Although in the martial arts coach education system, the static subject teaching knowledge modules are composed of the same knowledge components in the preservice and postservice martial arts coach training, due to the different teaching experience and personality differences of different martial arts coaches, they are faced with different teaching situations, and the different interaction between the static knowledge modules leads to the individual differences in the generated AI emotion analysis, which makes the martial arts coach profession have its irreplaceable high creative characteristics and also highlights the wisdom, interactive generation, and other characteristics of the martial arts coach work. On the background of the current application of artificial intelligence education, martial arts coaches are complex, open, and innovative compared to replacing artificial intelligence with the traditional closed, accurate, complex, and procedural problem-solving of martial arts coaches. We need to work on solving various problems. This complex and innovative problem-solving ability derives from the generation of wisdom of martial arts coaches based on the context of complex problems and the dynamic understanding and construction of the martial arts coach's static knowledge module at the root of wisdom. Coach generation lies in the generation and application of subjects that teach the knowledge of martial arts coaches that integrate artificial intelligence [26]. Therefore, the structure model of martial arts coaches integrating AI teaching knowledge (AI emotion analysis) is composed of static knowledge modules and includes the generation and application of personalized knowledge structure of martial arts coaches in different teaching situations.

The subject teaching knowledge of martial arts coaches combined with artificial intelligence is a kind of understanding of martial arts coach's teaching knowledge, students' learning knowledge, and the interaction between artificial intelligence teaching and learning knowledge based on students' all-round development in a specific teaching situation. This kind of knowledge is not only different from the knowledge of subject experts, but also different from the knowledge of curriculum experts and even different from the knowledge of artificial intelligence experts. It is the effective integration of these three knowledge modules by martial arts coaches in their specific teaching situations for students' allround development, and it is the key for martial arts teachers to use artificial intelligence to replace part of the work of martial arts coaches, so as to make teaching more effective and more conducive to promoting students' personalized learning and comprehensive development of knowledge.

\section{Results and Discussion}

4.1. Interaction Analysis of Emotion Improvement and Stability before Artificial Intelligence Martial Arts Competition. As shown in Figure 1, AI emotion analysis has interactive generative characteristics. Only by the presence and 


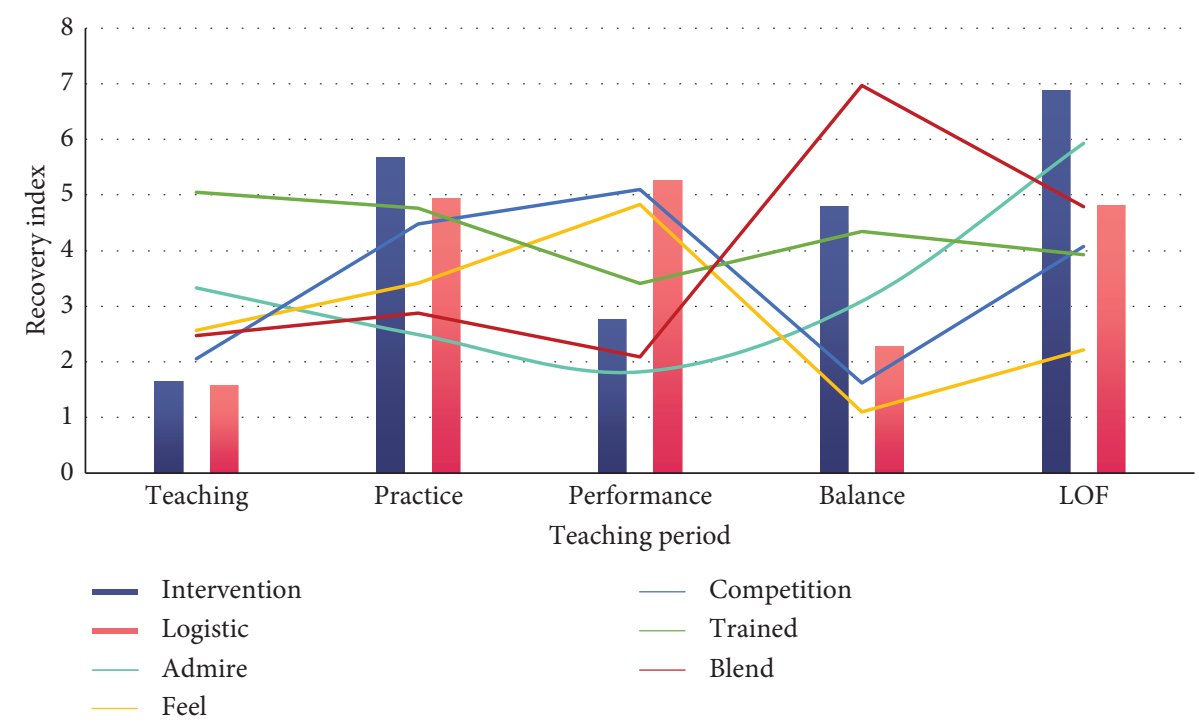

FIGURE 1: Interactive generative characteristics of artificial intelligence sentiment analysis.

development of the wisdom of martial arts coaches can we have the wisdom development of students, the active educational application of artificial intelligence, and promoting the effective development of wisdom education. AI emotion analysis does not only come from static knowledge module acquisition, but also come from the dynamic generation of martial arts coaches in specific teaching situations; from the knowledge taught by martial arts coaches, students' learning knowledge, and the interaction of AI teaching and learning knowledge in specific teaching situations artificial intelligence can be generated dynamically by the interaction of three elements. Therefore, AI sentiment analysis not only has its explicit and visible static knowledge components, but also has its implicit and tacit dynamic knowledge structure generation. At the same time, AI emotion analysis has individual characteristics.

Under the background of the current application of artificial intelligence education, the practical wisdom of martial arts coaches' AI emotional analysis should be improved by the effective exploration of the teaching practice of integrating artificial intelligence, so as to promote the transformation and construction of martial arts coaches' AI emotional analysis. As shown in Figure 2, the promotion of the practical wisdom of martial arts coaches' AI emotion analysis application not only needs the support of rich teaching practice experience, but also needs the in-depth reflection and reconstruction of martial arts coaches based on their own experience, and this reflection and reconstruction depends on the martial arts coaches' in-depth exploration of the teaching practice of integrating human intelligence.

As shown in Figure 3, in the training of on-the-job martial arts coaches, it is necessary to promote the dynamic understanding and construction of the subject teaching knowledge by the integration of artificial intelligence by the trained martial arts coaches based on the same course heterogeneity of artificial intelligence teaching. In the current postservice martial arts coach training, the subjectivity integration of theory and practice has shifted. Theory is the theory of college martial arts coaches, and practice is the practice of front-line martial arts coaches. After the trained martial arts coaches receive theoretical training, it is difficult for the theory to be transformed into the practice of the trained martial arts coaches. Therefore, in the training of onthe-job martial arts coaches, to promote the dynamic acquisition of the front-line martial arts coaches' teaching knowledge of integrating artificial intelligence, we should organize the trained martial arts coaches to carry out the same course heterogeneous teaching competition based on artificial intelligence teaching and employ the front-line excellent martial arts coaches and the experts of artificial intelligence education application in colleges and universities to carry out the corresponding analysis and comments, to help the trained martial arts coaches construct their dynamic knowledge structure integrating artificial intelligence.

As shown in Figure 4, when the model of martial arts tactics is 4, the AIC, BIC, and ABIC values are the minimum, and the LMR and Blrt values are statistically significant, indicating that the model data with four martial arts tactics fit well, and the entropy value of the four martial arts tactics is the largest. Therefore, C4 model is selected as the optimal model, and the conditional probability diagram is drawn. There were significant differences in the scores of three negative emotions and four aggressive behaviors among the four martial arts tactics.

As shown in Figure 5, the probability of attack behavior and negative emotion of martial arts tactics I is high, which is defined as "high aggressive and high negative emotion group," accounting for $15.4 \%$ of all subjects; the probability of martial arts tactics II in four kinds of aggressive behavior is high but in three kinds of negative emotion is low, which is defined as "high aggressive low negative emotion group," accounting for $21.8 \%$ of the total subjects; the probability of three kinds of negative emotions in martial arts tactics III is high, while the probability of four kinds of aggressive 


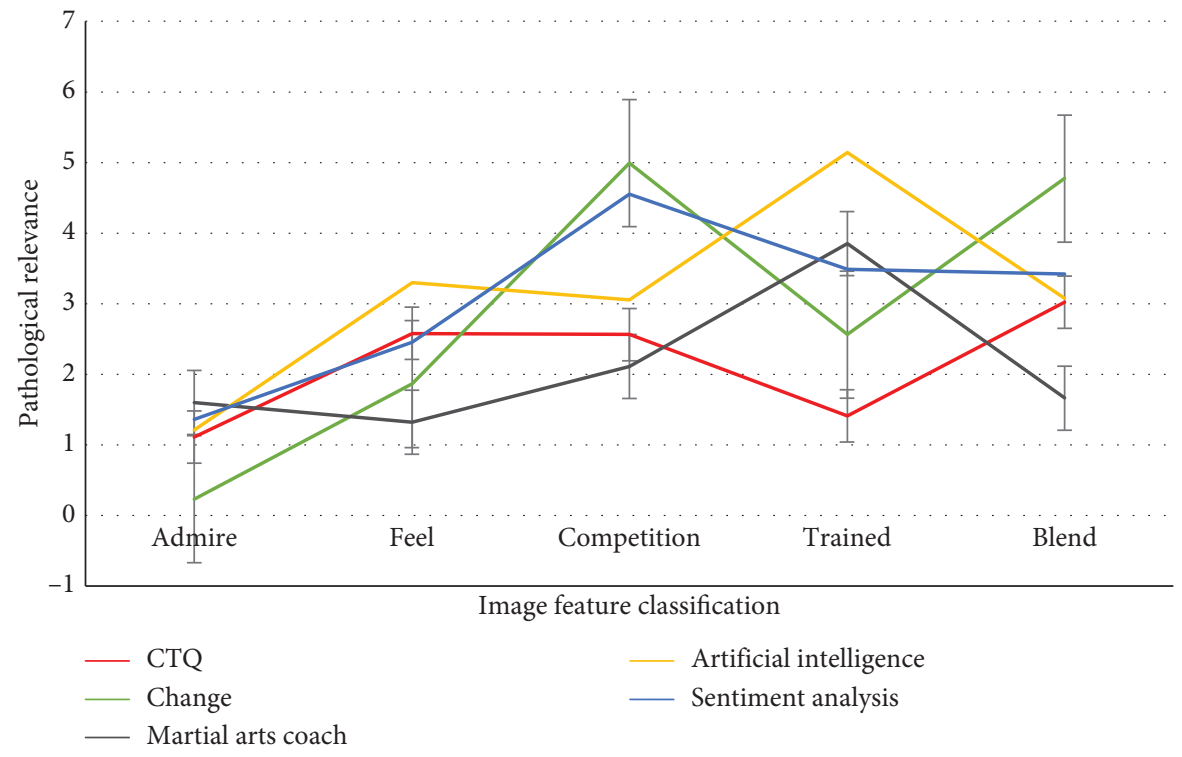

Figure 2: Artificial intelligence emotion analysis of martial arts coach.

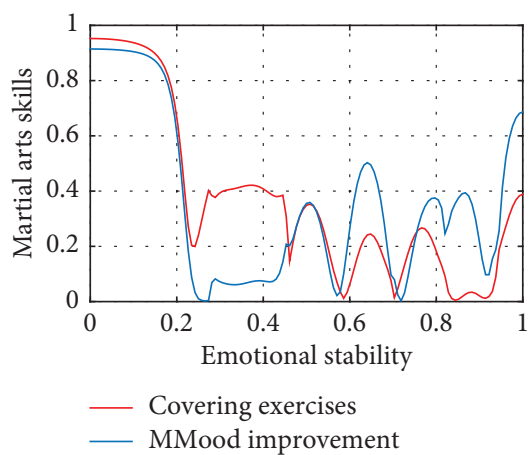

(a)

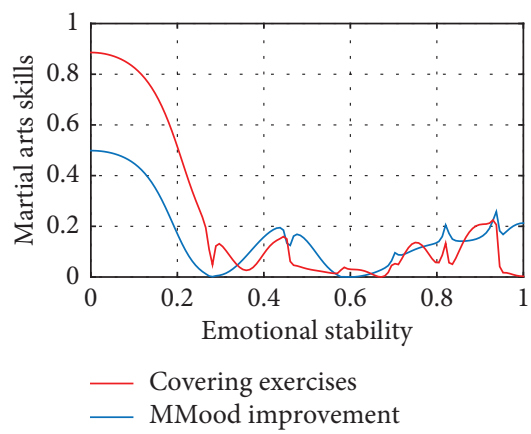

(d)

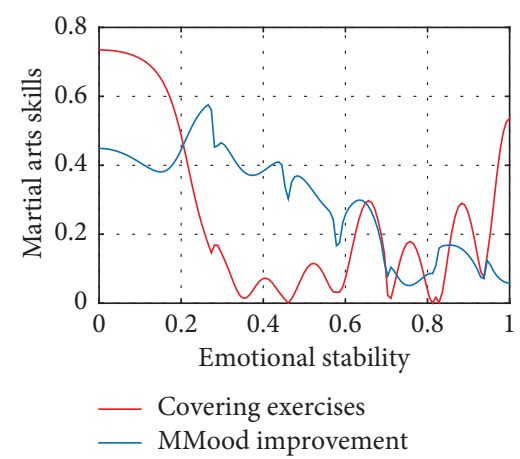

(b)

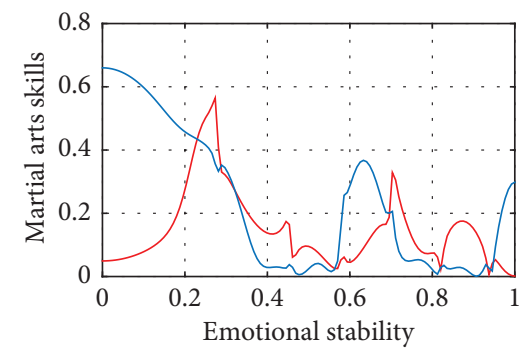

MMood improvement

(e)

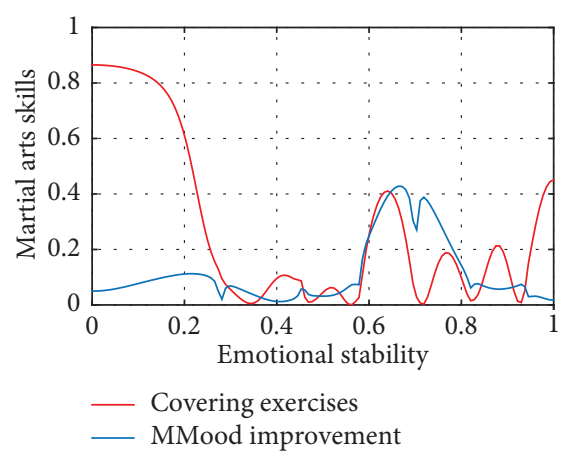

(c)

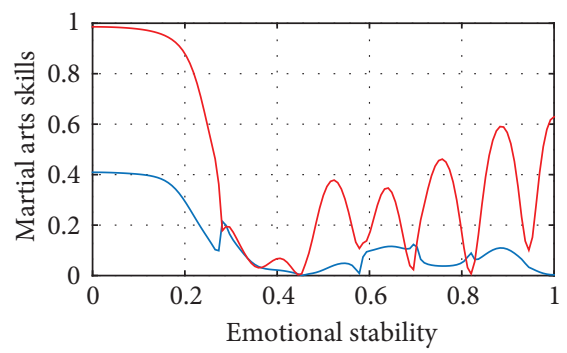

- Covering exercises

— MMood improvement

(f)

FIGURE 3: Heterogeneous promotion of the same class based on artificial intelligence teaching.

behavior is low. It is defined as "low aggressive and high negative emotion group," accounting for $10.25 \%$ of the total subjects.

As shown in Figure 6, the occurrence probability of aggressive behavior and negative emotion of martial arts tactics is far lower than the other three categories; this is defined as the "low aggression, low negative emotional group" and accounts for $53.3 \%$ of all subjects. Potential confounding factors with precompetitive trauma as the independent variable, emotional behavior groups as the dependent variable, and "low aggressive and low negative emotional groups" as references were analyzed by multiclass 


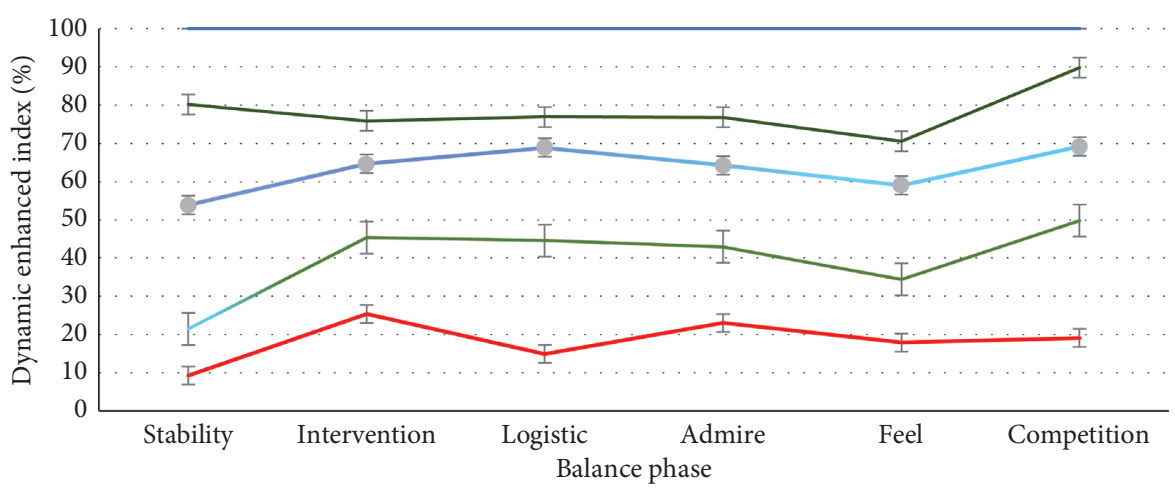

$\begin{array}{ll}\text { - Balance of power } & \text { - Teaching system } \\ \text { - Performance } & \text { - Mentor system }\end{array}$

- - Practice

Figure 4: C4 model and optimal model.

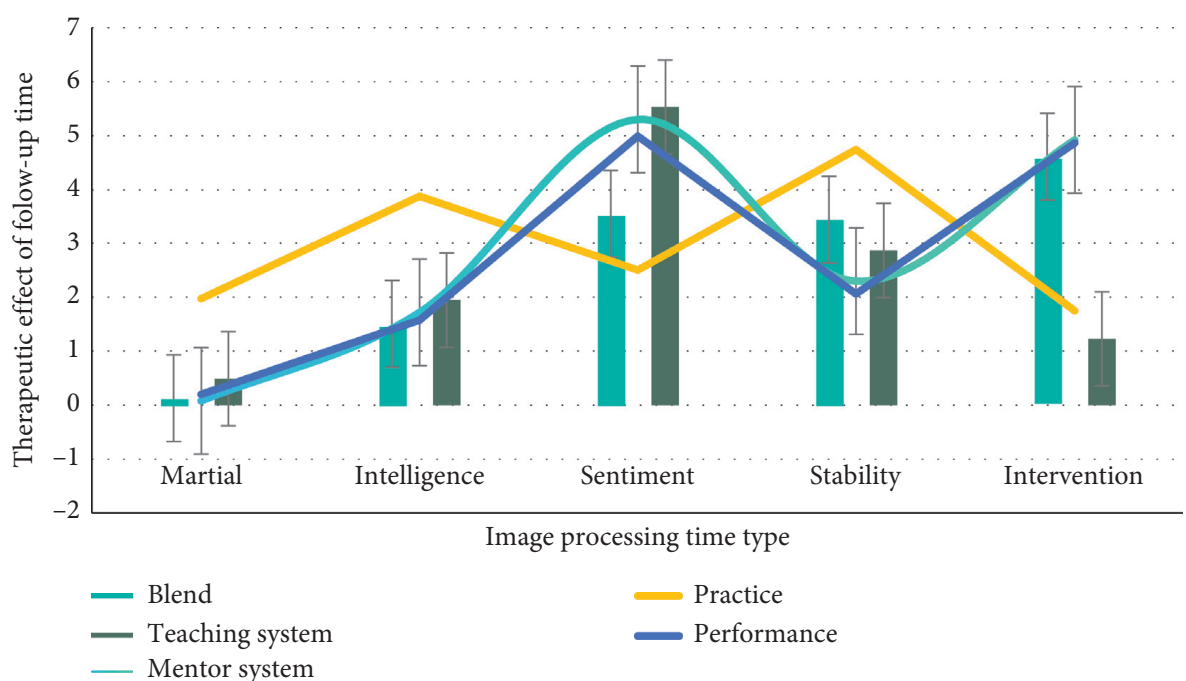

Figure 5: Probability of aggressive behavior and negative emotions.

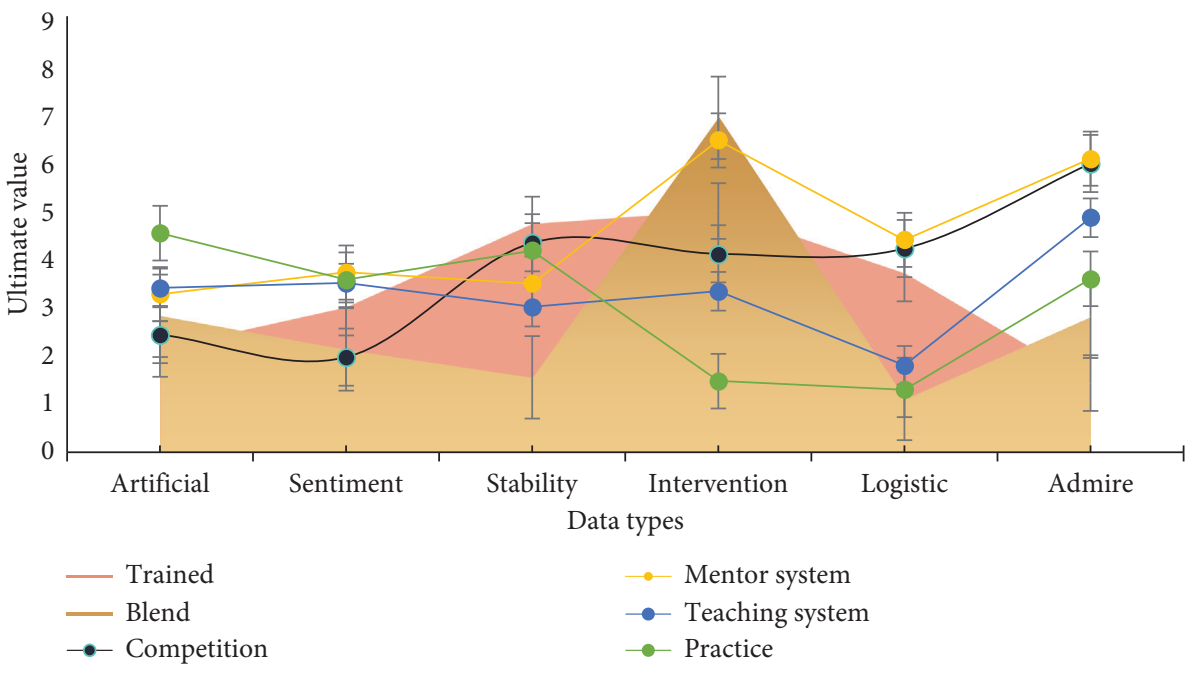

FIGURE 6: Probability of aggressive behavior and negative emotions. 
logistic regression. The goodness of fit of the four models as good.

As shown in Table 1, each martial arts coach has different professional growth experience and different hardware and software configuration of AI education applications in his school. Different martial arts coaches have different understanding of AI emotion analysis of martial arts coaches and correspondingly have different acceptance and recognition; therefore, it also has asynchronous AI emotion analysis application ability and practical intelligence generation. Therefore, based on the different professional development experience of each martial arts coach, he has the corresponding personalized AI emotion analysis, application ability, and practical wisdom generation. Under the background of the current application of artificial intelligence education, to ensure the effective transformation of martial arts coaches' subject teaching knowledge and the effective construction of martial arts coaches' AI emotion analysis, it is necessary not only to provide systematic theoretical courses for martial arts coaches from the outside, but also to ensure the acquisition of martial arts coaches' static subject teaching knowledge module integrating AI; it also needs to provide martial arts coaches with integrated teaching practice experience of integrating artificial intelligence to ensure the acquisition of the knowledge structure of dynamics science and education of martial arts coaches integrating artificial intelligence. It also needs martial arts coaches to enhance the practical wisdom of the application of artificial intelligence emotion analysis through effective exploration of teaching practice problems of integrating artificial intelligence. To promote the acquisition of martial arts coaches' static AI emotion analysis under the current background of AI education application, in order to ensure the acquisition of martial arts coaches' static AI emotion analysis, we should provide a static knowledge module for the generation of martial arts coaches' teaching knowledge with the new curriculum of martial arts coaches' education theory of preservice and postservice integration, to ensure that martial arts coaches master and acquire static knowledge modules, to lay a knowledge foundation for martial arts coaches to acquire dynamic knowledge structure.

As shown in Table 2, it should be based on the integration of artificial intelligence and virtual reality technology to promote the generation of teaching practice experience of martial arts coaches. The generation of dynamic knowledge structure requires the generation of direct education experience based on different teaching situations. Due to the unsophisticated teaching experience of quasi-martial arts coaches and new martial arts coaches, the inherent contradiction between the cultivation of novice martial arts coaches' teaching experience and the acquisition of students' high-quality education is due to the current contradiction between artificial intelligence and virtual education. It can be solved by the integration of augmented reality. Based on the support of artificial intelligence and virtual reality technology, quasi-martial arts coaches and novice martial arts coaches can have more diverse teaching experience, which is conducive to the effective acquisition of dynamic AI emotion analysis.
The new knowledge pattern based on the integration of the three knowledge modules will be incorporated into the overall curriculum of martial arts coach education, to ensure the mastery of integrated knowledge from quasi-martial arts coaches to in-service martial arts coaches. As shown in Table 3, in the new martial arts coach education theory curriculum, we should pay attention to the organic infiltration and integration of $\mathrm{AI}$ teaching and learning knowledge in the traditional martial arts coach teaching knowledge, students' learning knowledge, and other related martial arts coach education curricula. We can focus on the changes of martial arts coaches' teaching and students' learning caused by the integration of AI teaching and learning. The transformation of traditional martial arts coach teaching and students' learning caused by the integration of artificial intelligence should be included in the martial arts coach education theory curriculum system as an ad hoc course.

As shown in Figure 7, the knowledge of AI teaching and learning mainly refers to the education data layer, algorithm layer, perception layer, cognitive layer, and education application layer behind the AI education system, the education design logic, application logic, and interaction logic behind the AI education system, and the teaching advantages and disadvantages of the AI education system, and the design ethics and application ethics of AI education system. In addition to the three independent knowledge modules, AI sentiment analysis also includes new knowledge components generated from the integration of the three modules. Because of the integration of AI teaching and learning knowledge, the traditional martial arts coaches' knowledge about teaching has changed, which not only points to the general teaching knowledge of martial arts coaches, but also points to the integration of AI teaching and learning knowledge of martial arts coaches.

The first mock exam is divorced from any specific teaching situation and is difficult to generate. As shown in Figure 8, the teaching situation is not a static deterministic situation, but a complex and open generative situation. Therefore, AI emotion analysis is not a static knowledge module with its own boundaries, but a dynamic blending result of each knowledge module based on the specific teaching situation. Facing different teaching objects and fields, martial arts coaches with AI emotion analysis can determine whether to choose AI, what kind of AI to choose, how to use AI, and how to carry out their own teaching process based on AI teaching and learning, to better promote the all-round development of students. Secondly, the level of $\mathrm{AI}$ emotion analysis of martial arts coaches is in the process of integrated development from students' low-level cognitive development to high-level teaching and education based on specific teaching practices.

As shown in Figure 9, the fact and conceptual knowledge teaching of artificial intelligence develops procedural and metacognitive knowledge teaching, pays attention to the allround education penetration in knowledge teaching, and promotes the integration and all-round development process of students' high-level cognitive ability based on metacognition. At the same time, the application ability of AI 
TABLE 1: The generation of dynamic knowledge structure.

\begin{tabular}{lccccc}
\hline Item & CTQ & Change & Martial arts coach & Artificial intelligence & Sentiment analysis \\
\hline Admire & 1.11 & 0.23 & 1.6 & 1.21 & 1.36 \\
Feel & 2.58 & 1.86 & 1.32 & 3.3 & 2.45 \\
Competition & 2.56 & 4.99 & 2.11 & 3.05 & 4.55 \\
Trained & 1.41 & 2.56 & 3.85 & 5.14 & 3.49 \\
Blend & 3.02 & 4.77 & 1.66 & 3.08 & 3.42 \\
\hline
\end{tabular}

TABLe 2: Artificial intelligence and virtual reality technology.

\begin{tabular}{|c|c|c|c|c|c|c|c|}
\hline Item & Intervention & Logistic & Admire & Feel & Competition & Trained & Blend \\
\hline Teaching system & 1.65 & 1.58 & 3.33 & 2.57 & 2.06 & 5.05 & 2.47 \\
\hline Practice & 5.68 & 4.94 & 2.49 & 3.42 & 4.48 & 4.76 & 2.88 \\
\hline Performance & 2.77 & 5.26 & 1.82 & 4.83 & 5.1 & 3.41 & 2.09 \\
\hline Balance of power & 4.8 & 2.27 & 3.09 & 1.1 & 1.62 & 4.34 & 6.97 \\
\hline LOF & 6.88 & 4.81 & 5.93 & 2.22 & 4.07 & 3.93 & 4.79 \\
\hline
\end{tabular}

TABLe 3: New martial arts coach education theory curriculum setting.

\begin{tabular}{lccccc}
\hline Item & Blend & Mentor system & Teaching system & Practice & Performance \\
\hline Martial & 0.13 & 0.08 & 0.49 & 1.97 & 3.87 \\
Intelligence & 1.51 & 1.72 & 1.95 & 2.51 & 1.58 \\
Sentiment & 3.55 & 5.3 & 5.53 & 4.74 & 4.99 \\
Stability & 3.44 & 2.3 & 2.87 & 1.75 & 2.07 \\
Intervention & 4.61 & 4.92 & 1.23 & 4.86 \\
\hline
\end{tabular}

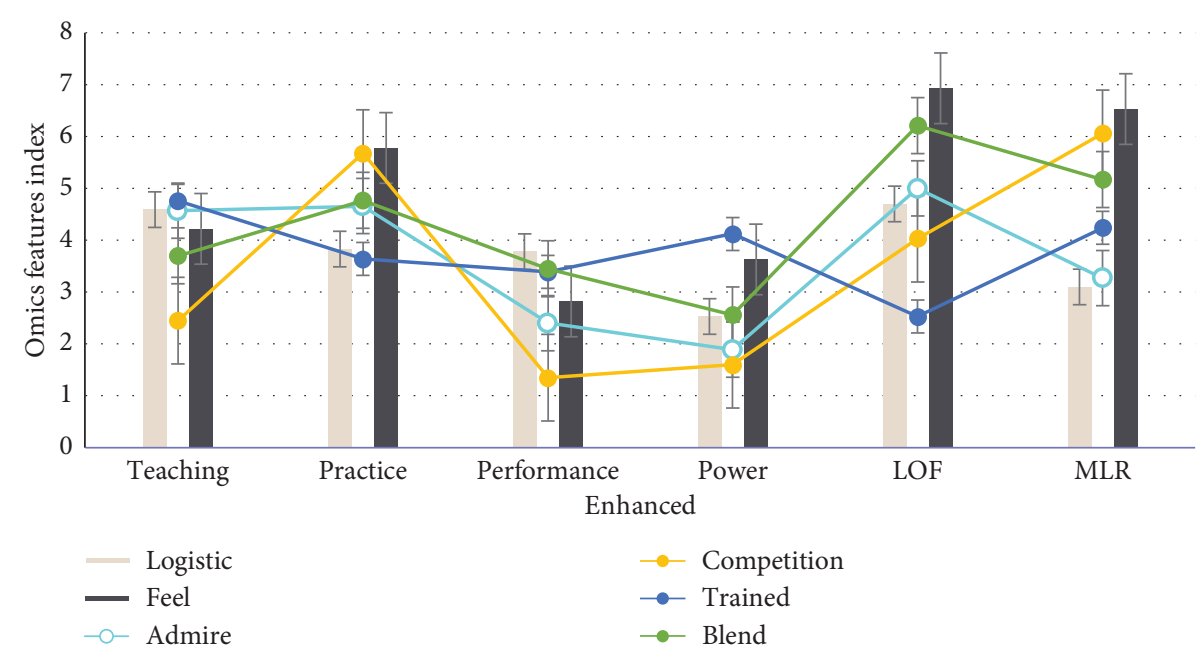

Figure 7: The knowledge of artificial intelligence teaching and learning.

emotion analysis of martial arts coaches is in the process of dynamic and personalized development based on different professional development stages of martial arts coaches. With the professional development of martial arts coaches, the application ability of AI emotion analysis of martial arts coaches is also in constant development, from the initial stage of recognition to gradual acceptance and then to continuous exploratory development.

Compared with no emotion analysis and on-the-sport guidance for martial arts athletes, this analysis and guidance method can effectively evaluate the emotion of martial arts athletes and give intervention measures as shown in Figure 10. Compared with the coach's intuitive guidance, the intervention measures in this paper increase the speed of $65 \%$. From the experimental object of this paper, athletes give the same level of corresponding emotional guidance suggestions when they have emotional fluctuations, which can improve the efficiency of athletes by more than $35 \%$ psychologically. Therefore, the use of artificial intelligence analysis methods can effectively improve the emotional 


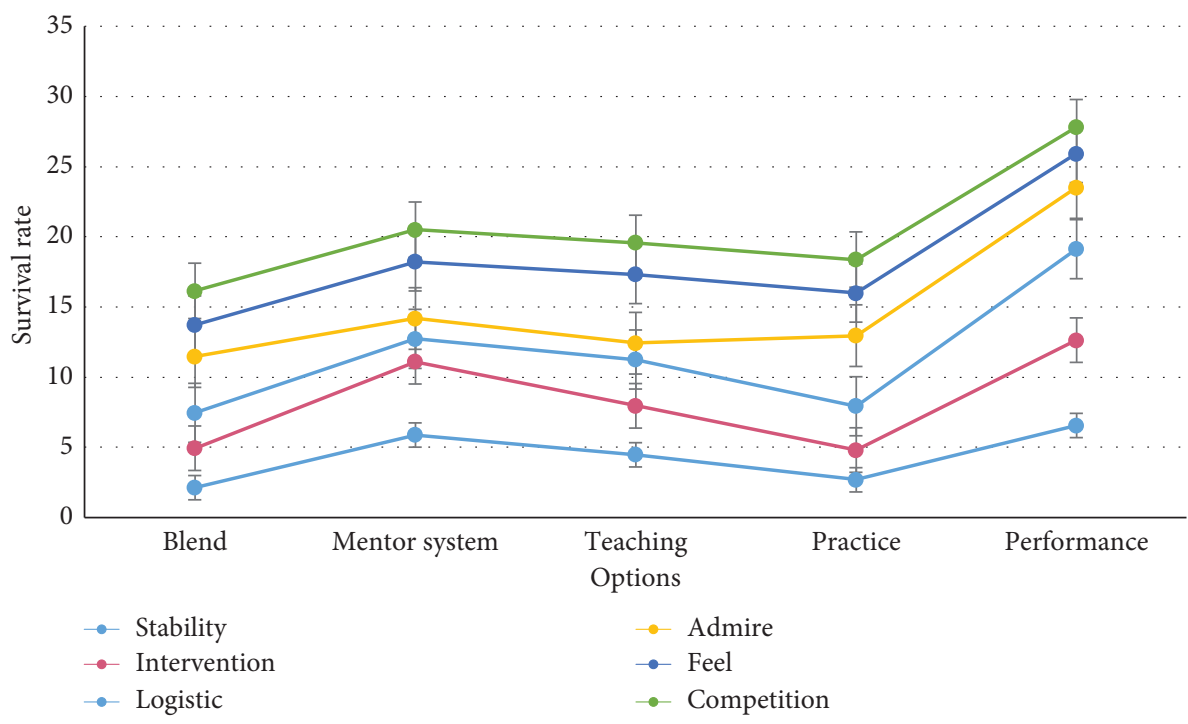

Figure 8: Complex and open generative situation.
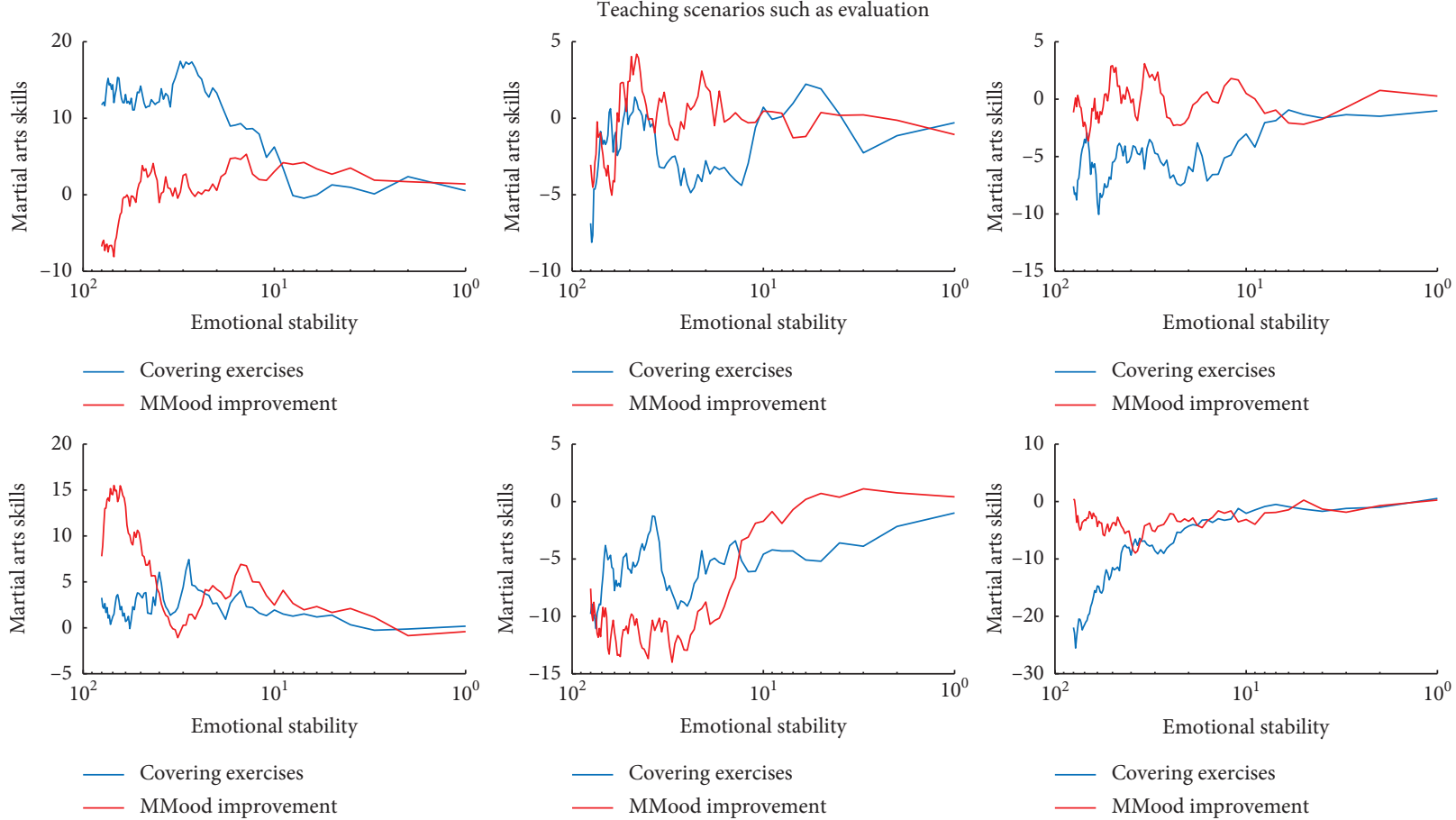

Figure 9: Professional development of different martial arts coaches.

stability of martial arts athletes before the game, so that they can deal with all kinds of emergencies on the spot.

4.2. Discussion. Due to the quasimartial arts coaching, quasiteaching, and virtual interactivity of artificial intelligence, the advancement of artificial intelligence education applications has caused a crisis between the traditional martial arts coach role and the martial arts coach career. The "theory of the demise of martial arts coaches" is on the rise, which forces us to reflect on the characteristics of martial arts coaches as a profession. As the inner foundation and basic guarantee of the professional development of martial arts coaches, the subject teaching knowledge of martial arts coaches is first faced with reflection and reconstruction. Therefore, this article attempts to explore the construction of the subject teaching knowledge of martial arts coaches integrating artificial intelligence based on the background of artificial intelligence education application. Under the current background of artificial intelligence education applications, artificial intelligence partially replaces the traditional subject knowledge education work of martial arts 


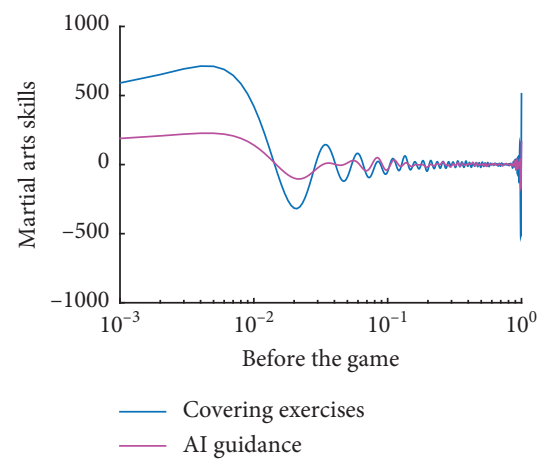

(a)

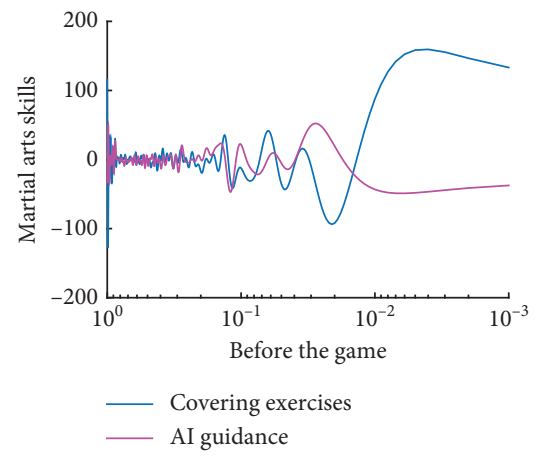

(d)

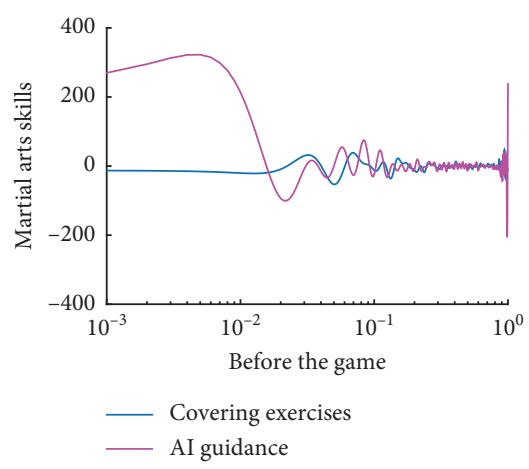

(b)

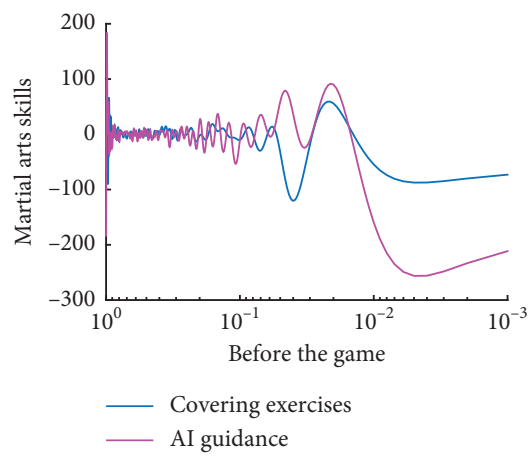

(e)

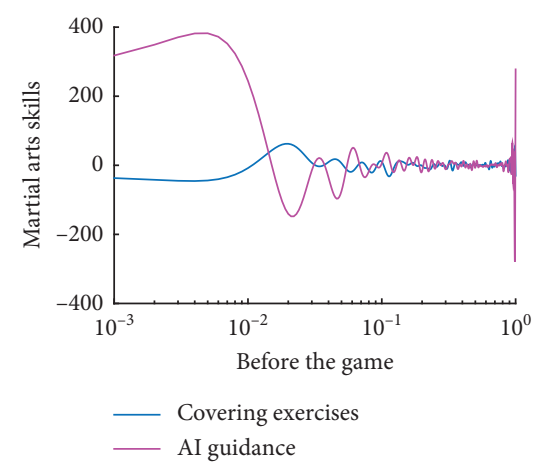

(c)

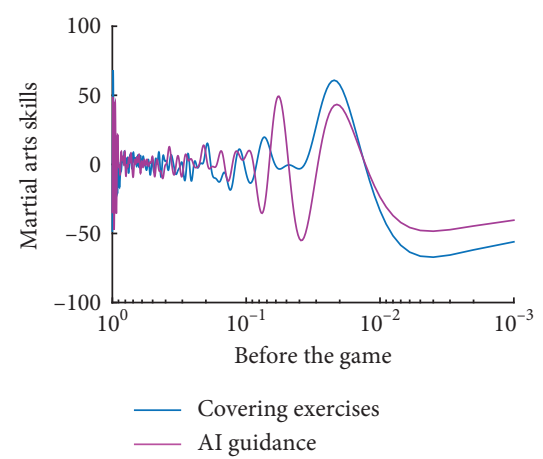

(f)

Figure 10: The rate of interventions in their methods.

coaches, and it is difficult to replace the subject education work, so the subject education knowledge component of martial arts coaches is facing an update and the dimension of martial arts coach education knowledge needs to be urgently expanded. The teaching knowledge level of martial arts coach subject needs to be upgraded. The current situation of the new era centered on the application of artificial intelligence education has pushed the teaching knowledge of martial arts coaches to begin to integrate artificial intelligence.

The connotation of the subject teaching knowledge of artificial intelligence (artificial intelligence emotion analysis) is due to the transfer of the traditional subject teaching knowledge of martial arts coaches to artificial intelligence under the current artificial intelligence education application background and the partial replacement and countermeasures of artificial intelligence for the teaching work of martial arts coach subject knowledge. Disciplinary education is hard to replace, and the teaching knowledge of martial arts coaches is transformed into the subject teaching knowledge of integrated artificial intelligence (artificial intelligence, emotion analysis). The martial arts coach education curriculum should be integrated based on multiple dimensions from knowledge to emotion to morality, to ensure the multidimensional mining and interpretation of artificial intelligence emotion analysis by prospective martial arts coaches and in-service martial arts coaches. That is to say, in the new martial arts coach education curriculum, in addition to mining the artificial intelligence emotional analysis curriculum content from the traditional single knowledge dimension, attention should also be paid to the interpretation of the emotional and moral dimensions; to help the quasi-martial arts coaches and martial arts coaches develop multidimensional artificial intelligence emotion analysis, interpretation and acquisition of knowledge of emotion to morality will help them master the relevant knowledge based on artificial intelligence teaching and learning to promote the development of students' cognition, emotion, and moral integrity.

\section{Conclusions}

Under the background of the current application of artificial intelligence in education, with the extensive and deep integration of artificial intelligence in the teaching field, it is necessary for traditional martial arts coaches to turn to the subject teaching knowledge of integrating artificial intelligence (artificial intelligence and emotion analysis). Artificial intelligence emotion analysis is a reconstruction of martial arts coaches' subject teaching knowledge beyond the traditional TPACK and ICT-pack technology perspective. It not only focuses on the technical components of subject teaching knowledge, but also pays more attention to the missing emotional and moral dimensions due to the partial substitution of artificial intelligence for martial arts coaches' knowledge teaching and pays more attention to the dynamic and personalized construction of martial arts coaches based on the situation. The construction of AI emotion analysis of martial arts coaches is conducive not only to establishing the integration and value guidance of martial arts coaches for AI 
teaching, but also to avoiding the arrogation of AI in the field of education and teaching. Only if the martial arts coaches themselves recognize the importance of transforming subject education knowledge and the martial arts coaches themselves focus on improving the practical wisdom of AI sentiment analysis applications will external forces be the driving force for their professional growth. Only in this way can the practical wisdom of martial arts coaches be promoted so that we can look back on the dilemma of educational practice integrated with $\mathrm{AI}$ and conduct education and research based on the reflection and dilemma. Therefore, the next step of this paper will verify the feedback mechanism of artificial intelligence combined with emotion analysis and pay attention to the fluctuation of martial arts on-the-spot emotion of scientific and moral level.

\section{Data Availability}

Data sharing is not applicable to this article as no datasets were generated or analyzed during the current study.

\section{Conflicts of Interest}

The authors declare that they have no conflicts of interest.

\section{Acknowledgments}

This work was supported by the project of young backbone teachers of Henan Polytechnic University (2018XQG-22).

\section{References}

[1] Y. Zheng, S. H. Ley, and F. B. Hu, "Global aetiology and epidemiology of type 2 diabetes mellitus and its complications," Nature Reviews Endocrinology, vol. 14, no. 2, pp. 88-98, 2018.

[2] J. Harreiter and M. Roden, "Diabetes mellitus definition, classification, diagnosis, screening, and prevention (update 2019)," Wiener klinische Wochenschrift, vol. 131, no. S1, pp. 6-15, 2019.

[3] R. A. Hackett and A. Steptoe, "Type 2 diabetes mellitus and psychological stress-a modifiable risk factor," Nature Reviews Endocrinology, vol. 13, no. 9, pp. 547-560, 2017.

[4] H. Abrahamian, A. Kautzky-Willer, A. Rießland-Seifert et al., "Mental disorders and diabetes mellitus (update 2019)," Wiener klinische Wochenschrift, vol. 131, no. S1, pp. 186-195, 2019.

[5] N. Sartorius, "Depression and diabetes," Body-Mind Interaction in Psychiatry, vol. 20, no. 1, pp. 47-52, 2018.

[6] A. Petersmann, D. Müller-Wieland, U. A. Müller et al., "Definition, classification and diagnosis of diabetes mellitus," Experimental and Clinical Endocrinology \& Diabetes: Official Journal, German Society of Endocrinology [and] German Diabetes Association, vol. 127, no. S1, pp. S1-S7, 2019.

[7] B. Arneth, R. Arneth, and M. Shams, "Metabolomics of type 1 and type 2 diabetes," International Journal of Molecular Sciences, vol. 20, no. 10, pp. 2467-2468, 2019.

[8] H. Bryan, "Leisure value systems and recreational specialization: the case of trout fishermen," American Journal of Veterinary Research, vol. 44, no. 5, pp. 861-864, 2020.

[9] C. M. Hägerhäll, T. Purcell, and R. Taylor, "Fractal dimension of landscape silhouette outlines as a predictor of landscape preference," Journal of Environmental Psychology, vol. 24, no. 4, pp. 247-255, 2020.

[10] D. E. Hand and R. D. Brown, "Enhancing visual preference of ecological rehabilitation sites," Landscape and Urban Planning, vol. 58, no. 1, pp. 57-70, 2020.

[11] Q. D. Huang, Q. Wu, G. X. Mao, S. Y Wang, B. B Jia, and G. F Wang, "Current status of forest medicine research in China," Biomedical and Environmental Sciences: BES, vol. 31, no. 7, pp. 551-554, 2018.

[12] N. J. Kuvaas, R. D. Dvorak, and M. R. Pearson, "Self- regulation and alcohol use involvement: latent class analysis," Addictive Behaviors, vol. 39, no. 1, pp. 146-152, 2019.

[13] J. Lee, B. J. Park, and Y. Tsunetsugu, "Effect of forest bathing on physio logical and psychological responses in young Japanese male subjects," Public Health, vol. 125, no. 2, pp. 93-100, 2021.

[14] M. Lewicka, "Place attachment: how far have we come in the last 40 years?" Journal of Environmental Psychology, vol. 31, no. 3, pp. 207-230, 2021.

[15] J. Maas, R. A. Verheij, and P. P. Groenewegen, "Green space, urbanity, and health: how strong is the relation?" Journal of Epidemiology \& Community Health, vol. 60, no. 7, pp. 587$592,2019$.

[16] R. L. Moore and A. R. Graefe, "Attachment to recreation settings: the case of rail trail users," Leisure Sciences, vol. 16, no. 1, pp. 17-31, 2020.

[17] S. W. O'Leary-Kelly and R. J. Vokurka, "The empirical assessment of construct validity," Journal of Operations Management, vol. 16, no. 4, pp. 387-405, 2020.

[18] J. F. Palmer, "Using spatial metrics to predict scenic perception in a changing landscape: dennis, Massachusetts," Landscape and Urban Planning, vol. 69, no. 2, pp. 201-218, 2020.

[19] K. K. Peschardt and U. K. Stigsdotter, "Associations between park characteristics and perceived restorativeness of small public urban green spaces," Landscape and Urban Planning, vol. 112, no. 2, pp. 26-39, 2019.

[20] H. M. Proshansky, A. K. Fabian, and R. Kaminoff, "Placeidentity: physical world socialization of the self," Journal of Environmental Psychology, vol. 3, no. 1, pp. 57-83, 2020.

[21] R. L. Ryan, "Exploring the effects of environmental experience on attachment to urban," Natural Areas. Environment and Behavior, vol. 37, no. 1, pp. 3-42, 2020.

[22] E. Sonntag-Öström, T. Stenlund, M. Nordin et al., ““Nature’ s effect on my mind"-patients' qualitative experiences of a forest-based rehabilitation programme," Urban Forestry \& Urban Greening, vol. 14, no. 3, pp. 607-614, 2019.

[23] K. A. Tamminen and P. R. E. Crocker, "“I control my own emotions for the sake of the team": emotional self- regulation and interpersonal emotion regulation among female highperformance curlers," Psychology of Sport \& Exercise, vol. 14, no. 5, pp. 737-747, 2019.

[24] M. Tveit, A. Ode, and G. Fry, "Key concepts in a framework for analyzing visual landscape character," Landscape Research, vol. 31, no. 3, pp. 229-255, 2020.

[25] S. Ivanaj, G.-B. Nganmini, and A. Antoine, "Measuring E-learners' perceptions of service quality," Journal of Organizational and End User Computing, vol. 31, no. 2, pp. 83-104, 2019.

[26] L. Zhihan, C. Dongliang, L. Ranran, and A. Ammar, "Artificial intelligence for securing industrial-based cyber-physical systems," Future Generation Computer Systems, vol. 117, pp. 291-298, 2021. 